\title{
Grand Canonical Model Predictions For Nuclear Fragmentation
}

\author{
C. B. Das ${ }^{1,2}$, S. Das Gupta ${ }^{1},{ }^{*}$ and B. K. Jennings ${ }^{3}$ \\ ${ }^{1}$ Physics Department, McGill University, Montréal, Canada H3A 2 T8 \\ 2 Variable Energy Cyclotron Centre, 1/AF Bidhannagar, Kolkata 700 064, India \\ ${ }^{3}$ TRIUMF, 4004 Wesbrook Mall, Vancouver, Canada V6T $2 A 3$
}

(December 22, 2018)

\begin{abstract}
The grand canonical ensemble has been used to make predictions for composite yields using simple models for nuclear fragmentation. While this gives correct model prediction for high energy collisions, it can give very inaccurate results at intermediate energy.
\end{abstract}

25.70.-z,25.75.Ld,25.10.Lx

A very simple but very popular model for nuclear multifragmentation is this: the nucleus is heated up and breaks up into many pieces (composites and new produced particles if the energy is sufficient) strictly according to phase space. This occurs in an expanded volume, about three or four times the normal volume. Population strictly according to phase-space implies chemical and thermal equilibrium. For simplicity, we will omit new particle production. In such cases the number of dissociating particles is fixed. Nonetheless in the past it has been customary for calculational simplicity to use the grand canonical ensemble to describe multifragmentation [1-3]. In such a model (GCM), the number of particles in the dissociating system is not constant, however one can arrange to have the average number correspond to the actual system. If one is in the classical regime (Fermi or Bose statstics degenerates into MaxwellBoltzmann limit) then the average yield of a composite in the ground state is given by

$$
\left\langle n_{i, j}(\text { ground })\right\rangle=e^{i \beta \mu_{z}+j \beta \mu_{n}} f_{i, j}
$$

where $i$ is the proton number, $j$ is the neutron number of the composite, $\mu_{z}$ is the proton chemical potential, $\mu_{n}$ is the neutron chemical potential and $f_{i, j}$ is given by:

$$
f_{i, j}=g \frac{V}{h^{3}}(2 \pi m T)^{3 / 2} a^{3 / 2} \exp \left(\beta E_{i, j}\right) .
$$

Here $V$ is the volume within which the particle moves, $a=i+j$ is the mass number of the composite, $m$ is the proton mass, $g$ is the spin degeneracy, $E_{i, j}$ is the binding energy of the composite and the Maxwell-Boltzmann distribution of the momentum of the particle has been integrated over. Usually populations into any states of the composite, ground and excited are included (a popular method of including the excited states is to use the Fermi-gas approximation) in which case $f_{i, j}$ is replaced by $\omega_{i, j}$, the one particle partition function of the particle. Thus we have

$$
\left\langle n_{i, j}\right\rangle=e^{i \beta \mu_{z}+j \beta \mu_{n}} \omega_{i, j}
$$

The Wigner-Seitz approximation of the coulomb energy is usually included [4] and this can be incorporated in the $\omega_{i, j}$ by replacing the coulomb self-energy $\frac{3 i^{2} e^{2}}{5 a}$ of the composite $a(=i+j)$ to $\frac{3 i^{2} e^{2}}{5 a}\left(1-\left(\rho / \rho_{0}\right)^{1 / 3}\right)$. The chemical potentials $\mu$ are fixed from

$$
\begin{aligned}
& \sum_{i, j} i\left\langle n_{i, j}\right\rangle=Z \\
& \sum_{i, j} j\left\langle n_{i, j}\right\rangle=N
\end{aligned}
$$

where $Z, N$ are the charge and neutron number of the dissociating system. The connection between the GCM and the Canonical Model (CM), as described in a textbook, is simple mathematics. Let us denote the CM partition function as $Q$ and the GCM partition function as $\tilde{Z}$, then

$$
Q_{n_{i, j}}=\frac{\left(\omega_{i, j}\right)^{n_{i, j}}}{n_{i, j} !} \text { and }
$$

${ }^{*}$ Corresponding author 


$$
\begin{aligned}
\tilde{Z} & =\prod_{i, j}\left[\sum_{n_{i, j}=0}^{\infty} e^{\left(i \beta \mu_{z}+j \beta \mu_{n}\right) n_{i, j}} Q_{n_{i, j}}\right] \\
& =\prod_{i, j} \exp \left[e^{\left(i \beta \mu_{z}+j \beta \mu_{n}\right)} \omega_{i, j}\right] .
\end{aligned}
$$
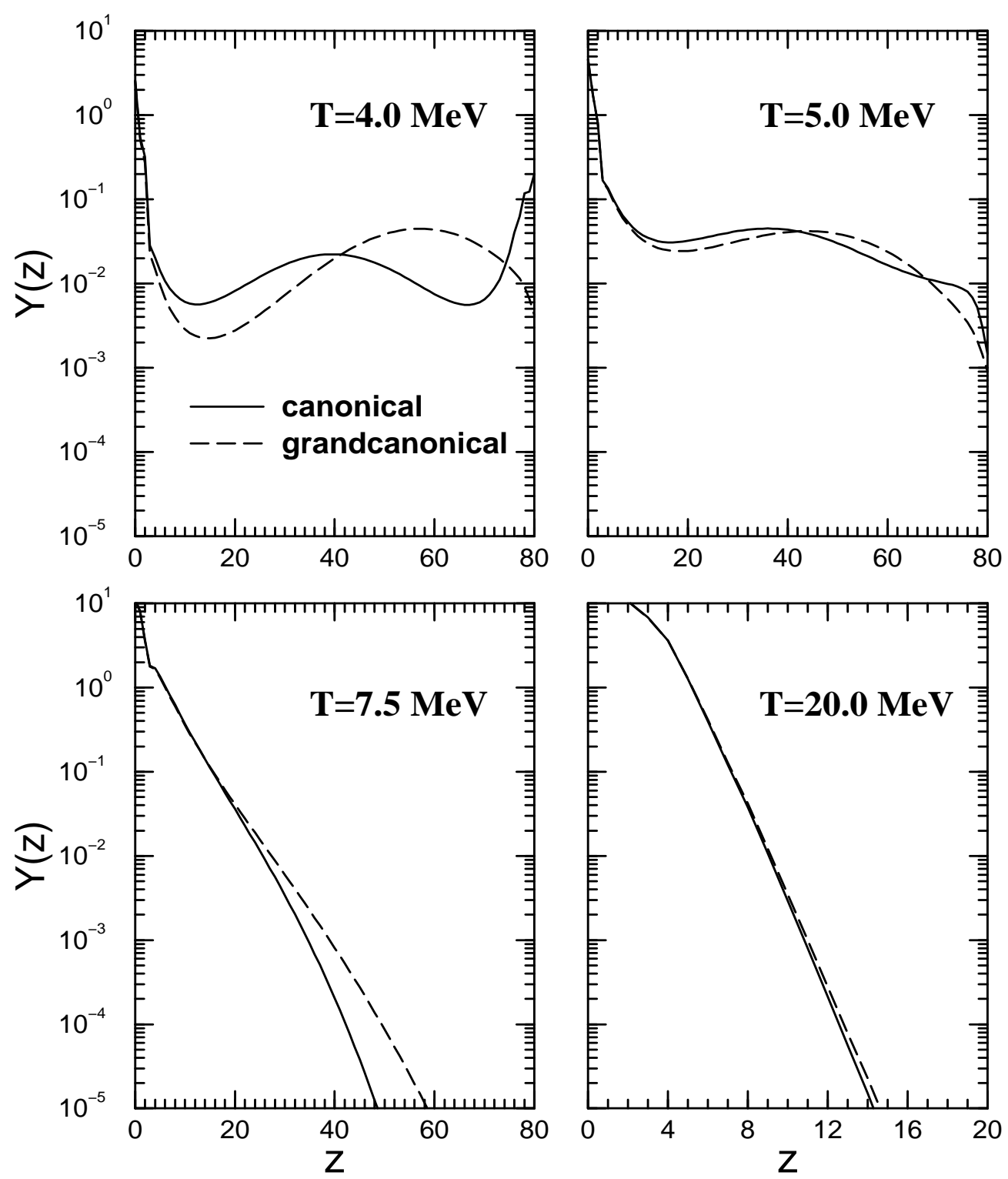

FIG. 1. The yields in the multifragmentation model using the Grand Canonical ensemble and the canonical ensemble for $A=200, Z=80$. Note that the two ensembles give very different values at $T=4 \mathrm{MeV}$.

Recently $[5,6]$ it has become possible to use the canonical model to calculate yields of fragmentation, whereas, in the past, the GCM was universally used. In the general case many composites are present and hence

$$
Q_{Z, N}=\sum \prod_{i, j} \frac{\omega_{i, j}^{n_{i, j}}}{n_{i, j} !}
$$

The sum is over all partitions of $Z, N$ into clusters and nucleons subject to two constraints: $\sum_{i, j} i n_{i, j}=Z$ and $\sum_{i, j} j n_{i, j}=N$. These constraints would appear to make the computation of $Q_{Z, N}$ prohibitively difficult which used to be the primary reason for the use of the grand canonical ensemble where with two constants $\mu_{z}, \mu_{n}$ one merely 
arranged the average values to be $Z$ and $N$. It has been recently realised that a recursion relation exists which allows the computation of $Q_{Z, N}$ quite easy on the computer even for large $Z$ or $N$ [7]. Three equivalent recursion relations exist, any one of which could be used. For example, one such relation is

$$
Q_{z, n}=\frac{1}{z} \sum_{i, j} i \omega_{i, j} Q_{z-i, n-j}
$$

The average number of particles of the species $i, j$ is given by

$$
\left\langle n_{i, j}\right\rangle=\omega_{i, j} \frac{Q_{Z-i, N-j}}{Q_{Z, N}}
$$

All nuclear properties are contained in $\omega_{i, j}$.
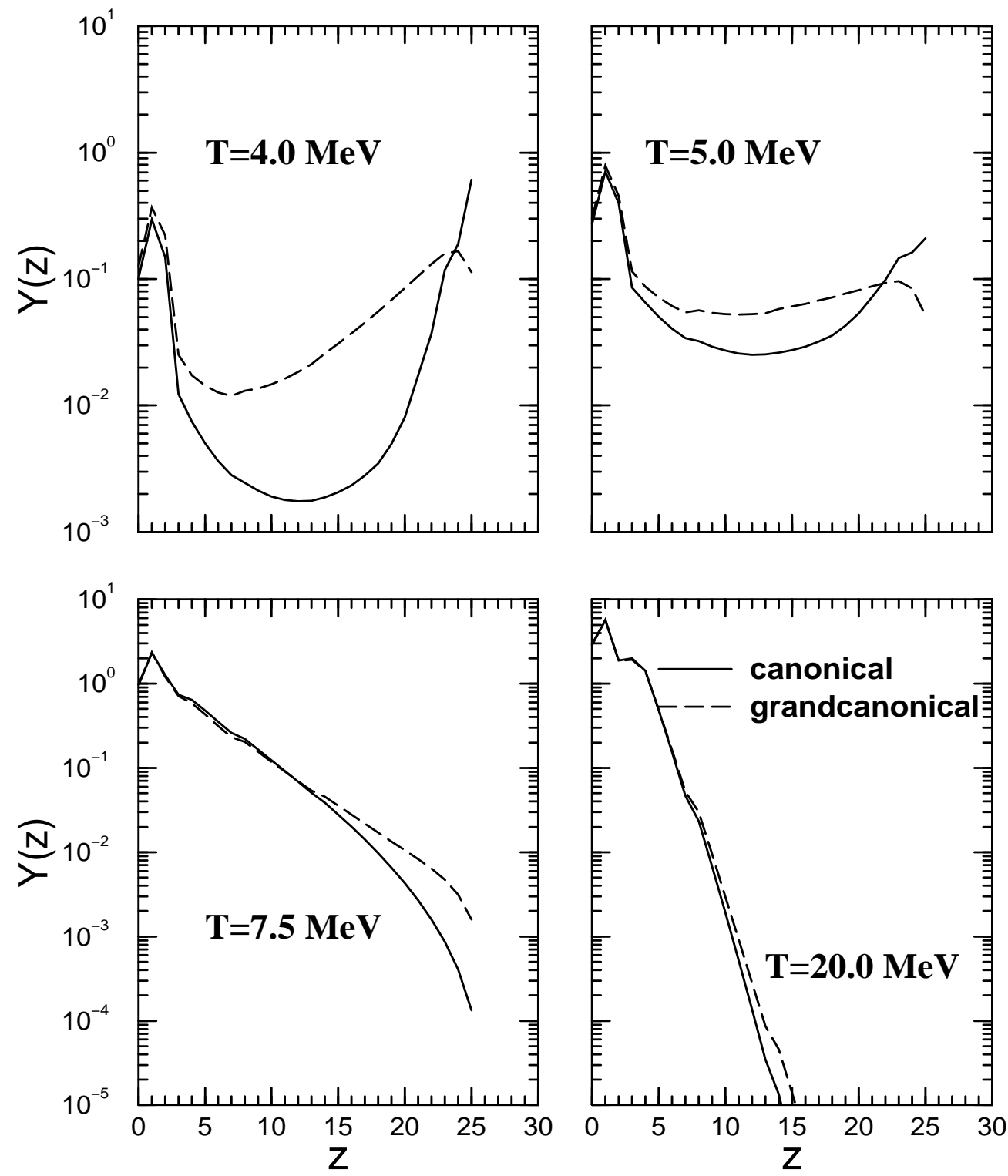

FIG. 2. The same as in Fig.1 except for the system $A=50, Z=25$. Again note the discrepancies at $T=4.0 \mathrm{MeV}$.

We are in a position to check, in the nuclear case, the grand canonical predictions for yields with the canonical values where particle number conservation is strictly enforced. We show this in Fig.1 for $A=200, Z=80, N=120$ (on the nuclear scale, a large system) and in Fig.2 for $A=50, Z=25, \mathrm{~N}=25$. As there are too many composites, 
we compare isotope yields (yields of the same $Z$ are added up and then compared). The GCM and CM predictions are quite close for high temperatures $(T \geq 20 \mathrm{MeV})$ but at low temperatures $(\approx 4 \mathrm{MeV})$ in spite of 200 being a large number, the GCM predictions are significantly different. One encounters such temperatures in intermediate energy heavy ion collisions thus one would conclude that one should not use the GCM in intermediate energies. GCM has been used at $100 \mathrm{MeV} /$ nucleon beam energy in the lab [8] where it may be just adequate but it has also been used at much lower energy $[9,10]$ where its usage is very questionable.

One has to ask if, when the two predictions differ, are both of them wrong or only one of them? If we consider thermal and chemical equilibrium to be the fundamental ingredient of the model, then the only way the present calculation in CM can be wrong is, if the approximation to quantum statistics (eq.3) is invalid around $5 \mathrm{MeV}$. Following a recent paper [11] we show this is not true and thus CM model results continue to be good. Here is a gist of the argument from that paper.

We use large volumes ( 3 or 4 times the normal volume). At low temperatures $(\approx 4 \mathrm{MeV})$ where one might imagine the approximation to fail, it survives because different composites appear thus there is not enough of any particular species to make (anti)symmetrisation an important issue. At much higher temperature, the number of protons and neutrons increase but as is well-known, the $n$ ! correction takes the approximate partition function towards the proper one. In a different world, the problem could get very difficult. Such a scenario would arise if the physics was such that at low temperatures we only had neutrons and protons and no composites. An even worse situation would arise if we had only neutrons (or protons). With these preliminaries, let us proceed to to estimate quantitatively the errors involved in actual cases that one might encounter. For simplicity only, in this section we will not put in excited states of composites and we have not put in the Wigner-Seitz correction although that could have been retained without much extra work.

A recursive relation similar to eq.4 exists even with incorporation of quantum statistics but $\omega_{i, j}$ 's are no longer one-particle partition functions. We illustrate this first with the example of only protons filling up orbitals $i, j, k \ldots$ in a box. Now

$$
\begin{aligned}
\ln Q_{g r}(\beta \mu) & =\sum_{i} \ln \left(1+e^{\beta \mu-\beta \epsilon_{i}}\right) \\
& =\sum_{i} \sum_{j} \frac{(-)^{j-1}}{j} e^{j\left(\beta \mu-\beta \epsilon_{i}\right)}
\end{aligned}
$$

The coefficient of $e^{\beta \mu k}$ is $x_{k}=\frac{(-)^{k-1}}{k} \sum_{i} e^{-k \beta \epsilon_{i}}$. The canonical partition function for $Z$ protons is very similar to eq.4:

$$
Q_{Z}(\beta)=\frac{1}{Z} \sum_{k=1}^{Z} k x_{k} Q_{Z-k}
$$

Here $Q_{0}$ is 1 . When the expressions for $x_{k}$ are used in the above equation, orbitals are given occupancies greater than one and then eliminated by subtraction. This can lead to severe round-off errors when applied to degenerate Fermi systems but will not affect the applications here. The number of protons $Z$ is given by

$$
Z=x_{1} \frac{Q_{Z-1}}{Q_{Z}}+2 x_{2} \frac{Q_{Z-2}}{Q_{Z}}+\ldots . . Z x_{Z} \frac{Q_{0}}{Q_{Z}}
$$

For generalisation, we will call $x_{k}$ above as $y_{1,0}^{[k]}$. The symbol 1,0 means it is a composite with 1 proton and 0 neutron. The symbol $k$ means it is obtained from the $k-t h$ term in the expansion; $y_{1,0}^{[k]}$ will contribute to $x_{k, 0}$.

If instead we had a boson, a deuteron, for example, we would have

$$
\begin{aligned}
\ln \left[Q_{\text {gr.can }}\left(\beta, \mu_{p}, \mu_{n}\right)\right] & =\sum_{i}-\ln \left(1-e^{\beta \mu_{p}+\beta \mu_{n}} e^{-\beta \epsilon_{i}}\right) \\
& =\sum_{i} \sum_{j} \frac{1}{j} e^{j\left(\beta \mu_{p}+\beta \mu_{n}-\beta \epsilon_{i}\right)}
\end{aligned}
$$

Thus in the case of deuterons $y_{1,1}^{[k]}$ (which would contribute to $x_{k, k}$ ) is given by $\sum_{i} \frac{1}{k} e^{-k \beta \epsilon_{i}}$.

We can treat an assembly of protons, neutrons, deuterons, tritons...etc. The recursive relation if the dissociating system has $Z$ protons and $N$ neutrons is 


$$
Q_{Z, N}=\frac{1}{Z} \sum_{i=1, Z, j=0, N} i x_{i, j} Q_{Z-i, N-j}
$$

The average number of a composite with $i_{1}$ protons and $i_{2}$ neutrons is given by

$$
<n_{i_{1}, i_{2}}>=y_{i_{1}, i_{2}}^{[1]} Q_{Z-i_{1}, N-i_{2}} / Q_{Z, N}+2 y_{i_{1}, i_{2}}^{[2]} Q_{Z-2 i_{1}, N-2 i_{2}} / Q_{Z, N}+\ldots
$$

Unless one is in an extreme degenerate fermi system, one can evaluate the $y$ factors by replacing sums with integration. For example, $y_{1,0}^{[n]}=\frac{(-)^{n-1}}{n} \sum_{i} e^{-n \beta \epsilon_{i}}$ where the sum is replaced by $\int e^{-n \beta \epsilon} g(\epsilon) d \epsilon=2 \frac{V}{h^{3}}\left(\frac{2 \pi m}{n \beta}\right)^{3 / 2}$. Here $V$ is the available volume. We have included the proton spin degeneracy; $m$ is the proton mass. For the deuteron, $y_{1,1}^{[k]}=$ $\frac{1}{k} \int e^{-k \beta \epsilon} g(\epsilon) d \epsilon$. This is $3 \times 2^{3 / 2} \frac{V}{h^{3}}\left(\frac{2 \pi m}{\beta}\right)^{3 / 2} \frac{e^{k \beta E_{b}}}{k^{5 / 2}}$ where $E_{b}$ is the binding energy of the deuteron. It is clear how to compute contributions from other composites.

We test the accuracy of the yields as calculated throughout the main text by comparing with a calculation where the complete theory of symmetrisation and antisymmetrisation is used. Subject only to the approximation that summation over discrete states has been replaced by an integration over a density of states, the calculation is exact. The results are taken from [11]. We take the dissociating system to have $Z=25$ and $N=25$. The lowest temperature considered is $3 \mathrm{MeV}$ (one might argue that at lower temperature a model of sequential decay is more appropriate). The highest temperature shown is $30 \mathrm{MeV}$. We take a freeze-out volume in which the composites can move freely in three times the volume of a normal nucleus with 50 nucleons. Aside from neutrons and protons we allow the possibility of composites. Spins and binding energies for deuteron, triton, ${ }^{3} \mathrm{He}$ and ${ }^{4} \mathrm{He}$ are taken from experiments. For higher mass composites the binding energy is taken from empirical mass formulas. For fermions, spin $1 / 2$ was assumed and for bosons spin 0 was assumed. For each $Z$ we take $N=Z-1, Z$, and $Z+1$. We present in the Table I. average yields of protons, neutrons, tritons, ${ }^{3} \mathrm{He},{ }^{4} \mathrm{He}$ and the sum of yields of all nuclei with charges greater than 12. Temperature range of 3 to $6 \mathrm{MeV}$ are of interest to many experiments. We also show the results at $30 \mathrm{MeV}$. The CM approximation for composites is seen to be quite good.

Granting that below a certain temperature, predictions from a grand canonical model gets to be quite erroneous, could one predict when it becomes bad and why? The answer to the first part is simple. Usually, the yield $\left\langle n_{z}\right\rangle$ (or $\left\langle n_{a}\right\rangle$ where $a$ is the mass number) falls with $z$ but below a certain value of the temperature, the yield, after falling initially, begins to rise again. If this happens, one must discard the GCM and do a CM. The rise of yields, after reaching a minimum, signifies several interesting features in intermediate energy multifragmentation models. In percolation and Lattice Gas model $[13,14]$ this signifies the appearence of a percolating cluster. In thermodynamic model, the temperature at which this rise, after the minimum, just disappears signifies a first order phase transition (in the infinite system and no coulomb limit) [5]. It suggests that at this temperature a large blob of the system, usually identified as a liquid, has just disappeared. It has been shown that at the transition temperature, the specific heat at constant volume is very different in GCM and CM although they match admirably at higher temperature [15]. The reason for the discrepancy is an unusually large fluctuation in the number of particles in the GCM below the transition temperature. We have however no fundamental understanding why such large fluctuations appear in the GCM below the transition temperature.

This work is supported in part by the Natural Sciences and Engineering Research Council of Canada.

[1] A. Z. Mekjian, Phys. Rev. Lett. 38, 640 (1977)

[2] J. Gosset, J. I. Kapusta, and G. D. Westfall, Phys. Rev. C18,844(1978)

[3] S. Das Gupta and A. Z. Mekjian, Phys. Rep.72, 131 (1981)

[4] J. P. Bondorf, A. S. Botvina, A. S. Iljinov, I. N. Mishustin, and K. Sneppen, Phys. Rep. 257, 133 (1995)

[5] S. Das Gupta and A. Z. Mekjian, Phys. Rev C57, 1361 (1998)

[6] P. Bhattacharyya, S. Das Gupta and A. Z. Mekjian, Phys. Rev C60, 054616 (1999)

[7] K. C. Chase and A. Z. Mekjian, Phys. Rev C50, 2078 (1994)

[8] J. Randrup and S. E. Koonin, Nucl. Phys. A356, 223 (1981)

[9] S. Pal, S. K. Samaddar, A. Das and J.N. De, Nucl. Phys. A586, 466 (1995)

[10] C. B. Das, A. Das, L. Satpathy and M. Satpathy, Phys. Rev. C 53, 1833 (1996)

[11] B. K. Jennings and S. Das Gupta, Phys. Rev. C 62, 014901(2000)

[12] S. Das Gupta, A. Z. Mekjian and M. B. Tsang, Advances in Nuclear Physics, vol.26, 91 (2001)

[13] W. Bauer, Phys. Rev. C 38, 1297 (1988) 
[14] J. Pan and S Das Gupta, Phys. Rev. C 51, 1384 (1995)

[15] C. B. Das, S. Das Gupta and A. Z. Mekjian, Phys. Rev. C 68, 031601(R), (2003)

TABLE I. Comparision of claculations of average yields and E/A. By exact we mean a calculation with proper symmetry. Sum over discrete orbitals in a box has been replaced by integration as is the usual practice.

\begin{tabular}{|c|c|c|c|c|c|c|c|c|c|}
\hline 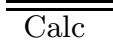 & $\overline{p p}$ & $\bar{n}$ & $\overline{\bar{d}}$ & $\bar{t}$ & ${ }^{3} \mathrm{He}$ & ${ }^{4} \mathrm{He}$ & $\overline{Z Z>12}$ & $\overline{\overline{\text { Temp. }} .}$ & $\overline{\overline{E / A}}$ \\
\hline approx & 0.307 & 0.032 & 0.050 & 0.007 & 0.054 & 0.679 & 0.945 & $3 \mathrm{MeV}$ & $-7.863 \mathrm{MeV}$ \\
\hline exact & 0.306 & 0.031 & 0.051 & 0.007 & 0.053 & 0.696 & 0.945 & $3 \mathrm{MeV}$ & $-7.861 \mathrm{MeV}$ \\
\hline approx & 1.174 & 0.898 & 1.177 & 0.560 & 0.641 & 2.489 & 0.051 & $6 \mathrm{MeV}$ & $-4.117 \mathrm{MeV}$ \\
\hline exact & 1.117 & 0.856 & 1.195 & 0.553 & 0.638 & 2.573 & 0.050 & $6 \mathrm{MeV}$ & $-4.135 \mathrm{MeV}$ \\
\hline approx & 4.127 & 3.955 & 4.812 & 2.099 & 2.052 & 1.985 & 0.000 & $12 \mathrm{MeV}$ & $4.401 \mathrm{MeV}$ \\
\hline exact & 3.860 & 3.696 & 4.941 & 2.090 & 2.051 & 2.021 & 0.000 & $12 \mathrm{MeV}$ & $4.308 \mathrm{MeV}$ \\
\hline approx & 10.937 & 10.893 & 7.664 & 1.686 & 1.650 & 0.379 & 0.000 & $30 \mathrm{MeV}$ & $28.914 \mathrm{MeV}$ \\
\hline exact & 10.512 & 10.468 & 7.885 & 1.732 & 1.696 & 0.395 & 0.000 & $30 \mathrm{MeV}$ & $28.844 \mathrm{MeV}$ \\
\hline
\end{tabular}

